\title{
Análise físico-química da água de três poços subterrâneos no município de Mossoró-RN
}

\author{
Rafael Barbalho de Lima* (Graduando em Química na Universidade do Estado do RN - UERN)
}

Daniele da Silva Oliveira (Mestranda do PPGCN na Universidade do Estado do Rio Grande do Norte UERN)

Rafael Oliveira Batista (Prof. Ad. Do DCAT e do PPMSA na Universidade Federal do Semi-Árido UFERSA)

Sandra Maria Campos Alves (Profa. Do do Instituto Federal de Educação, Ciência e Tecnologia do Rio Grande do Norte - IFRN)

Francisco Uberlanio Silva (Mestrando do Curso petróleo e gás da Universidade Potiguar - UNP)

Luiz Di Souza (Prof. Ad. do Dq e do PPGCN na Universidade do Estado do Rio Grande do Norte UERN)

*rafaelbarbalho.12@hotmail.com

\begin{abstract}
Resumo: A água é um recurso fundamental para a existência da vida. O planeta Terra está inundado de água com um volume de aproximadamente 1,4 bilhão de $\mathrm{km}^{3}$ cobrindo cerca de $71 \%$ da superfície da Terra. Apesar disso, muitas localidades ainda não têm acesso a água com características de potabilidade adequadas às necessidades do consumo. O município de Mossoró-RN localizado no semiárido nordestino, tem 70 \% de sua população abastecida por águas de poços subterrâneos. A qualidade da água para o consumo humano é de extrema importância e de modo geral as águas naturais de superfícies ou subterrâneas, não atendem aos padrões de potabilidade recomendados para consumo, necessitando de tratamento e controle de qualidade. A falta de saneamento básico e a exploração acima do aceitável são fatores que contribuem para a contaminação de mananciais subterrâneos, sendo estes maximizados por fatores naturais que diminuem a disponibilidade de água como a seca que atinge a região durante cinco anos. Este trabalho objetivou analisar as amostras de água subterrâneas coletadas em três diferentes pontos de Mossoró-RN, bem como comparar os valores obtidos em cada ponto entre si e com a legislação em vigor, avaliando a sua qualidade e as influências antrópicas do local nestas características. O município de Mossoró-RN está localizado no oeste do estado do Rio Grande do Norte, segundo o censo do IBGE, 2010, tem uma população de 259.886 habitantes. Foram coletadas amostras em três poços com profundidade aproximada de 90 metros. A amostra 1 foi coletada no bairro Nova Betânia (Campus da UNP), amostra 2 foi coletada no centro de Mossoró-RN (Rebouças Supermercado) e a amostra 3 foi coletada no bairro Alto de São Manoel (Residencial do Sr. Fausto Guilherme). Os resultados mostraram que as águas analisadas se enquadram na classe 3. Algumas características como sólidos dissolvidos, sulfato e nitrito atendem ao padrões da resolução CONAMA $n^{\circ} 396 / 2008$, outros como nitrato e especificamente o cloreto da amostra 2 demonstraram valores acima do permitido, evidenciando que os pontos 2 e 3 possuem um baixo serviço de esgotamento sanitário e uma densidade populacional maior, o que caracteriza esse fato.
\end{abstract}

Palavras-chaves: Qualidade; água; indicadores. 


\section{I NTRODUÇÃO}

A água é um recurso fundamental para a existência da vida. Nosso planeta está inundado de água com um volume de aproximadamente 1,4 bilhão de $\mathrm{km}^{3}$ cobrindo cerca de $71 \%$ da superfície da Terra. Apesar disso, muitas localidades ainda não têm acesso a água com características de potabilidade adequadas às necessidades do consumo humano (GRASSI, 2001).

Sabe-se que do volume total da água do planeta, a presença de água salgada, nos oceanos e mares, corresponde a 97,5 \% e a de água doce, em rios, lagos, geleiras e subsolo, corresponde apenas $2,5 \%$. Desta pequena quantidade de água doce, 68,9 \% encontra-se em geleiras e coberturas permanentes de neve, 29,9 \% é relacionado à água doce subterrânea e 0,3 \% são das águas dos rios e lagos (ALMEIDA, 2010).

Como consequência da explosão demográfica e do rápido aumento das necessidades da agricultura e da indústria moderna, os recursos hídricos constituem objeto de uma demanda crescente. Dessa forma é necessário preservá-la e utilizá-la racionalmente (BECKER, 2008; RAHMAN, et al., 2011; FERREIRA; ROCHA; FIGUEIREDO, 2015).

O Brasil é privilegiado com $12 \%$ da água doce superficial no mundo. Mais de $90 \%$ do território brasileiro recebe precipitações pluviométricas abundantes durante o ano e as condições climáticas e geológicas propiciam a formação de uma extensa e densa rede de rios, com exceção do semiárido, onde os rios são temporários, o que caracteriza um forte problema de distribuição e disponibilidade geográfica da água (REBOUÇAS et al., 1999; INSTITUTO SOCIO AMBIENTAL, 2015).

A água é um recurso imprescindível, sobretudo em regiões semiáridas afetadas por regime de precipitação pluviométrica irregular (VÖRÖSMARTY et al., 2010; JACOBSEN et al., 2012; van VLIET et al., 2013). Como é o caso da região Nordeste do Brasil, no qual está inserido o Rio Grande do Norte. A reduzida disponibilidade natural aliada à má gestão dos recursos hídricos propicia a contaminação das águas, comprometendo, ainda, mais o abastecimento público, acarretando em sérios riscos à saúde das populações e aos ecossistemas (MAFFEI et al., 2009).

O Nordeste semiárido é uma região pobre em volume de escoamento de água dos rios, situação explicada em função da variabilidade temporal das precipitações pluviométricas e das características geológicas dominantes, onde há predominância de solos rasos, baseados sobre rochas cristalinas e, consequentemente, baixas trocas de água entre o rio e o solo adjacente (CIRILO, 2008). Na Região Nordeste reservatório de águas subterrâneas possui cerca de 19,5 bilhões de metros cúbicos de água por ano, equivalente a 40 vezes o volume explorado no ano de 2006 (SOARES et al., 2006).

Nesta região são perfurados poços desde o início do século passado e a utilização da água subterrânea cresceu vertiginosamente e de forma descontrolada, em função das demandas de água nessa região e a falta de fontes superficiais na mesma (SAMPAIO; FEITOSA, 2014).

Um problema que deve ser destacado é a poluição dessas águas, a poluição das águas subterrâneas se concentra mais em áreas urbanas, onde se encontram grandes volumes de fontes de poluição, como esgotos não tratados e aterros comuns.

O contaminante inorgânico de maior preocupação em águas subterrâneas é o íon nitrato $\left(\mathrm{NO}_{3}{ }^{-}\right.$ ), que normalmente ocorre em aquíferos de zonas rurais e suburbanas. O nitrato em águas subterrâneas origina-se, principalmente, de quatro fontes: aplicação de fertilizantes com nitrogênio orgânico, inorgânico e de esterco animal em plantações; cultivo do solo; esgoto humano depositado em sistemas sépticos inapropriados e deposição atmosférica (BAIRD; CANN, 2011).

Exemplo claro dessa situação são os municípios de Natal e Mossoró no Estado do Rio Grande do Norte, basicamente abastecidas com água subterrâneas. Em Natal, onde o problema é mais bem estudado são 159 poços, dentre os quais 15 já deixaram de ser explorados por ruptura de revestimento e pela presença de nitrato em nível superior ao permitido pela Organização Mundial de Saúde - OMS. Somente $26 \%$ do esgoto é coletado por rede, e apenas $22 \%$ do esgoto coletado é tratado (BORSOI et al., 2002).

Em Mossoró e região o problema, ainda, não foi estudado em profundidade mais trabalhos já indicam uma situação, no mínimo preocupante, já que a situação do saneamento básico não é boa; em 2007 96,82 \% da população era atendida pelo abastecimento de água, e 28,26 \% da população foi 
atendida pelo esgotamento sanitário. A cidade é abastecida por 15 poços artesianos (água subterrânea do aquífero Açu), além de poços semi artesianos e até mesmo por cacimbões nas áreas rurais. Como consequência disso tem-se constatado poluição dos poços subterrâneos que abastecem as populações, especialmente pelo contaminante nitrato e aumento dos níveis de salinidade (BAIRD; CANN, 2011; PROGRAMA ÁGUA AZUL, 2011).

Outro grave problema é a seca prolongada na região, no Rio Grande do Norte muitos municípios já se encontram em estado de emergência, com barragens secas e açudes bem abaixo de sua capacidade. Na região do Seridó muitas cidades estão sendo abastecidas por carros pipas. No campo a situação se agrava mais ainda, onde a população não tem como manter seu gado, devido ao longo tempo de estiagem (SILVA, 2015).

Portanto, objetivou-se neste trabalho analisar as amostras de água subterrâneas coletadas em três diferentes pontos da cidade de Mossoró-RN, bem como comparar os valores obtidos em cada ponto entre si e com a legislação em vigor, avaliando a sua qualidade e as influências antrópicas do local nestas características.

\section{METODOLOGI A}

\subsection{Coleta e análise das amostras}

O município de Mossoró-RN está localizado no oeste do estado do Rio Grande do Norte, segundo o censo do IBGE, 2010, possui uma área de $2.099 \mathrm{~km}^{2}$ e, tem uma população estimada para o ano de 2015 de 288.162 habitantes. A cidade se encontra inserida no semiárido nordestino e tem 70 \% de sua população abastecida por águas de poços subterrâneos. As amostras foram coletadas em três poços com profundidade aproximada de 90 metros. A amostra 01 foi coletada no bairro Nova Betânia (Campos UNP), amostra 02 foi coletada no centro da cidade (Rebouças Supermercado) e a amostra 03 foi coletada no bairro Alto de São Manoel (Residencial do Sr. Fausto Guilherme). A Figura 1 ilustra os locais no momento em que foram feitas as coletas.

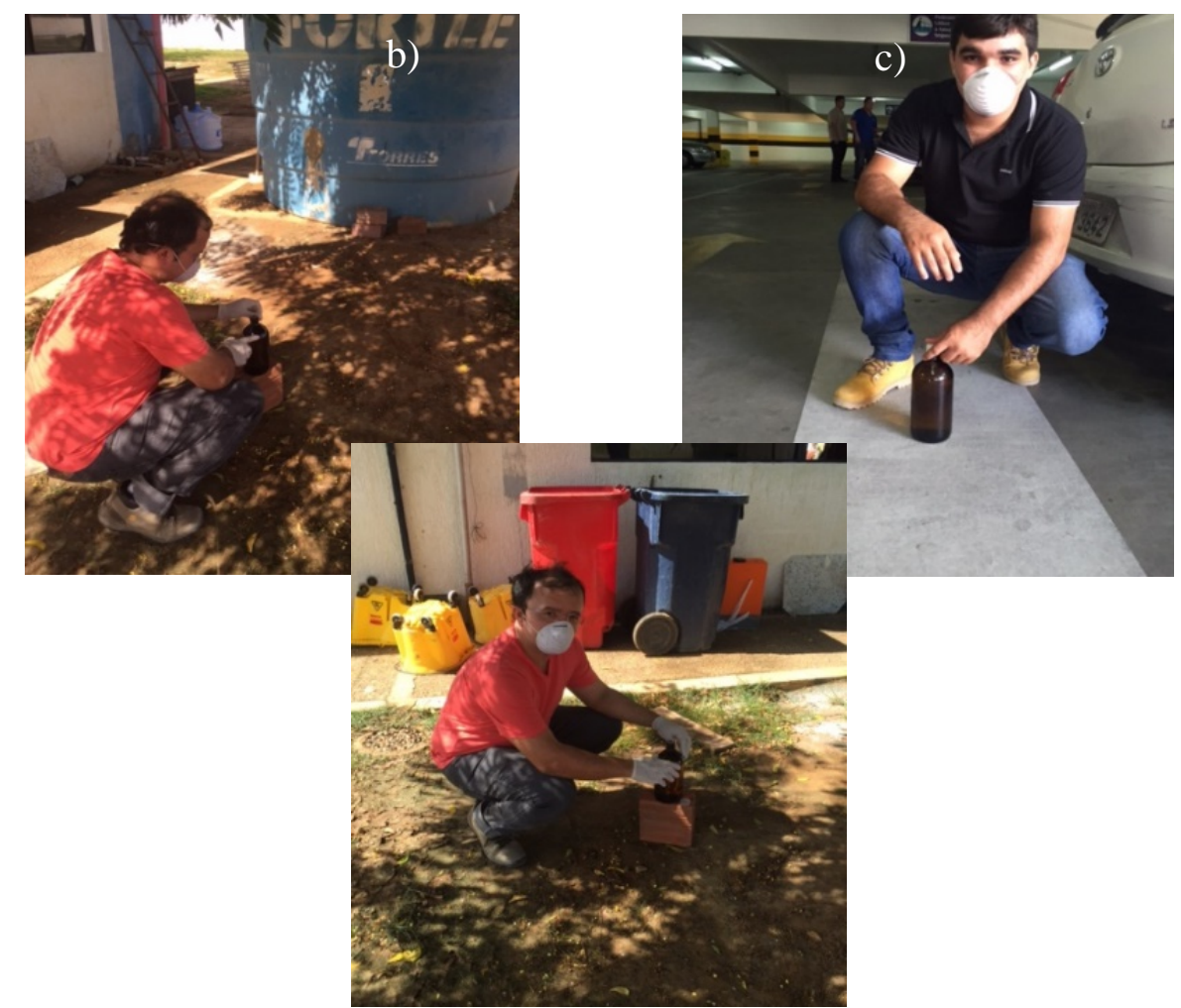

Figura 1. Local de coleta das águas subterrâneas a) UNP9), b) supermercado Rebouças no centro e c) Residência do Sr. Fausto Guilherme no bairro alto de São Manoel. 
As amostras foram coletadas de acordo com a normalização vigente em frascos âmbar, previamente limpos e imediatamente resfriadas e transportadas para o Laboratório de Catálise, Ambiente e Materiais (LACAM) pertencente ao Departamento de Química da Universidade do Estado do Rio Grande do Norte-UERN, onde as análises físico-químicas foram realizadas usando as metodologias prescritas no Standard Methods for the Examination of Water and Wastewater (APHA, 2005). As análises feitas foram: sólidos dissolvidos, nitrato, nitrito, amônio, nitrogênio total, cloreto, alcalinidade, salinidade, cálcio, dureza, magnésio, sulfato e fosfato. Todas as analisem foram feitas em triplicata e o resultado expresso como a média dessas medidas

\section{RESULTADOS E DISCUSSÃO}

Todos os resultados obtidos são apresentados no Quadro 1.

Quadro 1. Resultados das características físico-químicas encontradas para os três pontos de coleta.

\begin{tabular}{|c|c|c|c|c|}
\hline \multirow{3}{*}{$\begin{array}{r}\text { Coordenadas } \\
\text { Geográficas }\end{array}$} & \multicolumn{3}{|c|}{ Pontos de coleta } & \multirow{3}{*}{$\begin{array}{l}\text { Resolução } \\
\text { CONAMA } \\
n^{0} 396 / 2008\end{array}$} \\
\hline & 1 (UNP) & 2 (Rebouças) & 3 (Sr.Fausto) & \\
\hline & 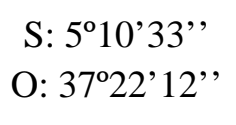 & $\begin{array}{l}\text { S: 5011’09', } \\
\text { O: } 37^{\circ} 20^{\prime} 29^{\prime},\end{array}$ & $\begin{array}{l}\text { S: 5012’33', } \\
\text { O: } 37^{\circ} 20^{\prime} 47^{\prime \prime}\end{array}$ & \\
\hline Sólidos dissolvidos (ppm) & 416 & 924 & 716 & 1000 \\
\hline Amônia (ppm) & 0,0216 & 0,01969 & 0,1443 & - \\
\hline Cloreto (ppm) & 84 & 266 & 126 & 250 \\
\hline Alcalinidade (ppm) & 87 & 158 & 138 & - \\
\hline Salinidade (ppm) & 138,43 & 438,37 & 207,65 & \\
\hline Fósforo (ppm) & 0,03158 & 0,04055 & 0,03062 & - \\
\hline Sulfato (ppm) & 9,13 & 184,77 & 55,83 & 250 \\
\hline Dureza (ppm) & 171,972 & 273,132 & 384,4 & - \\
\hline Cálcio (ppm) & 12,825 & 14,43 & 16,03 & - \\
\hline Magnésio (ppm) & 2,9 & 4,86 & 7,78 & - \\
\hline Nitrato (ppm) & 12,182 & 61,354 & 64,075 & 10,000 \\
\hline Nitrito (ppm) & 0,0022 & 0,0259 & 0,2271 & 1,000 \\
\hline Nitrogênio total (ppm) & 16,73 & 62,928 & 71,721 & - \\
\hline
\end{tabular}

Os resultados obtidos foram comparados com os prescritos na resolução CONAMA ${ }^{\circ}$ 396/2008 que dispõe sobre a classificação e diretrizes ambientais para o enquadramento das águas subterrâneas, as amostras analisadas se enquadram, de acordo com esta resolução na classe 3. Os valores de sólidos dissolvidos, sulfato e nitrito estão dentro dos limites permitidos nesta resolução. Os valores de nitrato e cloreto da amostra 2 se encontram acima do permitido e indicam a necessidade de um melhor monitoramento e intervenção das autoridades competentes. Resultados semelhantes têm sido encontrados em outros pontos da região como, em poços das bacias hidrográficas Piranhas-Açu, Apodi-Mossoró, Pirangi, Potengi, Doce, Ceará-mirim, Jacu, Faixa Litorânea Leste de Escoamento Difuso e Faixa Litorânea Norte de Escoamento Difuso. Esses poços apresentaram concentração do nitrato acima do limite aceitável e são utilizados para fins diversos, incluindo o consumo humano (PROGRAMA ÁGUA AZUL, 2011).

No Gráfico 1 e Quadro 1 podem-se observar os resultados obtidos para a característica nitrato, percebe-se que os valores de nitrato para todas as amostras excederam o valor máximo permitido, tendo valores maiores nas amostras 2 e 3 . Isso pode estar relacionado a falta de saneamento nessas regiões, pois os resultado apresentaram menor no ponto 1 , o mesmo é um local menos povoado da 
cidade e que por ser mais novo tem um bom serviço de saneamento básico; enquanto os pontos 2 e 3 se encontram em áreas mais antigas da cidade, aonde ainda existe deficiência no esgotamento sanitário e a densidade populacional é maior. Neste caso a área menos atendida por serviços de saneamento seria o bairro do alto de são Manoel (ponto 3) o que corresponde à realidade constatada por efluentes a céu aberto verificados em vários locais dessa região.

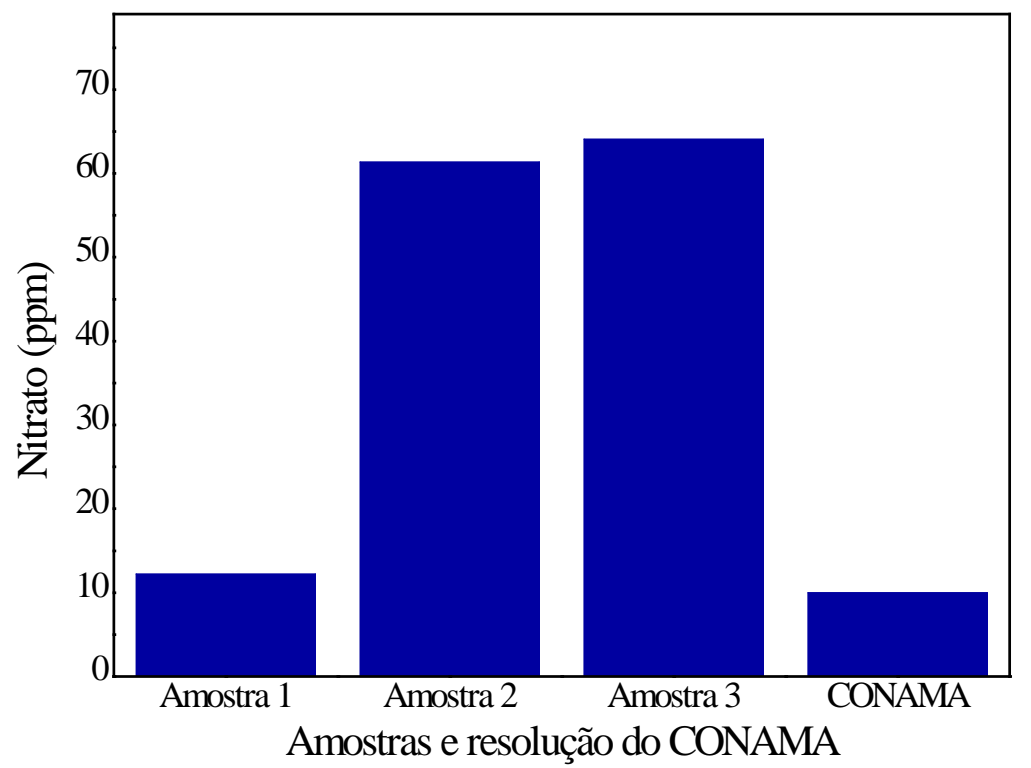

Gráfico 1- Resultados de nitrato para as três amostras analisadas e limite permitido na resolução do CONAMA.

O Gráfico 2 e Quadro 1, mostram os resultados obtidos para a característica cloreto. As amostras 1 e 3 possuem valores abaixo do permitido pela CONAMA e a amostra 2 excede o valor permitido para o consumo humano. Este resultado é mais um forte indicio de que a poluição é resultante da contaminação por esgotos sanitários por que este íon está fortemente presente na urina dos seres humanos. Observe que neste caso, a área de maior concentração de pessoas é a área que apresenta poluição com esta característica. Dejetos humanos e de animais possuem teor elevado de cloreto, devido ao cloreto de sódio ser um ingrediente comum nas dietas passando inalterado pelo sistema digestivo. Nas estações de abastecimento de águas, a presença de concentrações anormais de cloreto e material nitrogenado é um indício possível desse tipo de poluição. A concentração de cloreto em esgoto doméstico varia entre 30 e 100 ppm. Água com concentração muito elevada de cloreto causa danos em superfícies metálicas, em estruturas de construção e muitas espécies de plantas. A tolerância dos seres humanos para o cloreto nas zonas áridas, pode chegar a 900 ppm sem nenhum efeito fisiológico adverso. Para indivíduos acostumados a baixas concentrações, um alto teor de cloreto na água ingerida pode ter efeito laxativo (CETESB, 2010). 


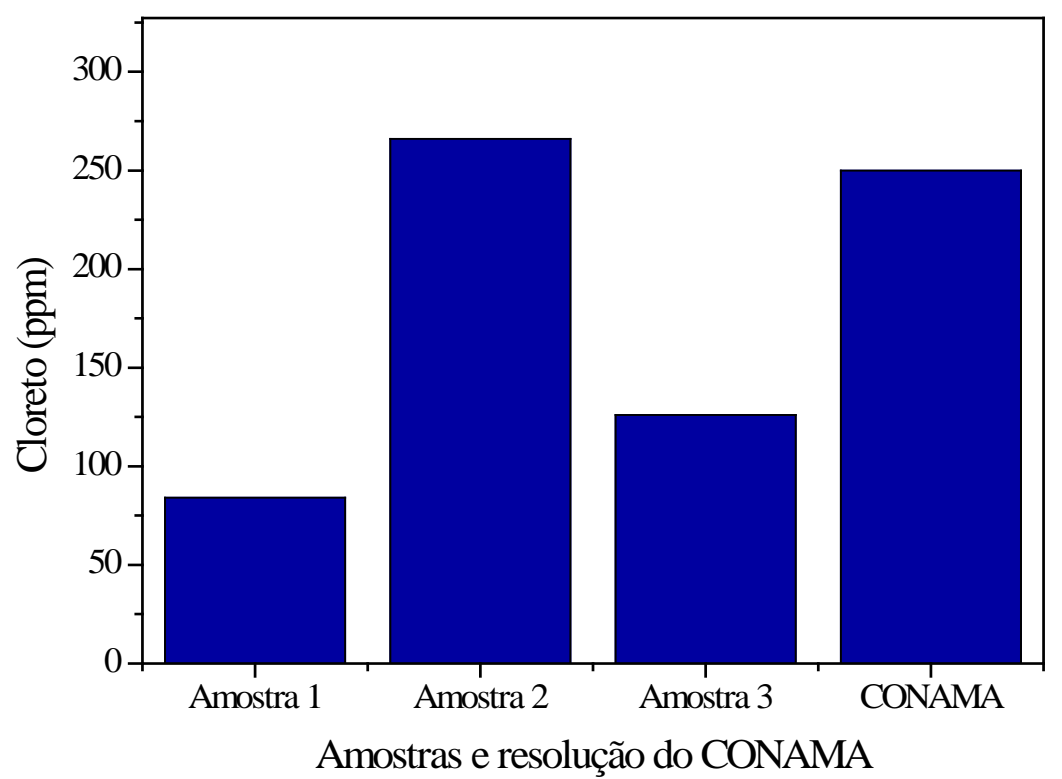

Gráfico 2- Resultados de cloreto para as três amostras analisadas e limite permitido na resolução do CONAMA.

Visando entender melhor o efeito da localização (espacialidade) nos resultados analisaram várias outras propriedades cujos limites não estão regulamentados pela legislação, mas são importantes para entender o problema. De forma dois comportamentos básicos foram observados para as análises apresentadas no Quadro 1. O primeiro comportamento valido para as propriedades sólidos dissolvidos, amônia, cloreto, alcalinidade, salinidade, fósforo e sulfato é representado pela concentração de sulfato no Gráfico 3, apresentam um aumento do ponto da UNP para o ponto do centro de Mossoró-RN e depois o valor diminui para o ponto do Alto de São Manoel, comportamento que assim como para o nitrato estaria relacionado a densidade populacional desses bairros, sendo que a maior produção de esgotos resultaria na elevação da concentração destes poluentes. Observe que todas estas variáveis estão fortemente ligadas a atividade de limpeza e higiene das pessoas e casas, sendo que alcalinidade, salinidade, fósforo e sulfato estão mais intimamente relacionados a limpeza (uso de detergentes e outros produtos de limpeza e higiene) e amônia, nitrito e cloreto estão mais relacionados com a questão do uso de produtos de higiene pessoal e de necessidades básicas.

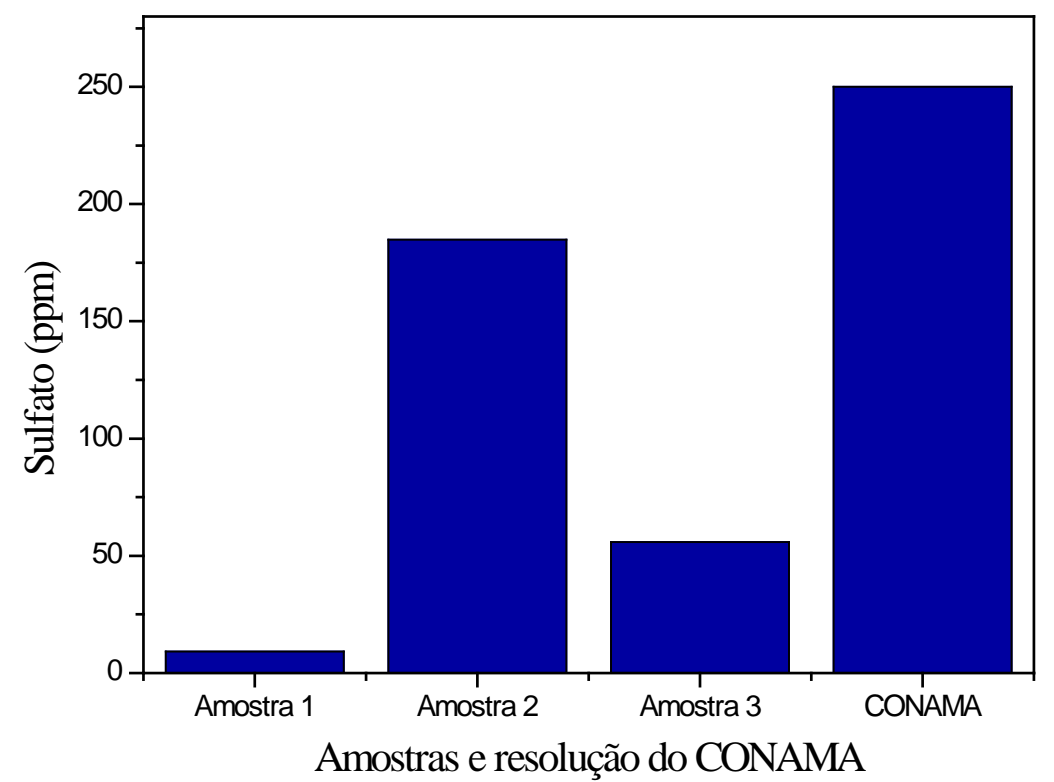

Gráfico 3- Resultados de sulfato para as três amostras analisadas e limite permitido na resolução do CONAMA. 
O outro comportamento valido para as propriedades dureza, cálcio, magnésio, nitrato, nitrito e nitrogênio total representado pela concentração da dureza (Gráfico 4 e Quadro 1), apresenta constante aumento do ponto UNP para o ponto centro de Mossoró-RN e para o ponto de Alto de São Manoel, fato que pode ser explicado pela maior densidade populacional, e pela falta de esgotamento sanitário, devido a isso há um aumento de concentração desses elementos. Quanto à qualidade das águas investigadas para fins de consumo humano, não existe, até o presente, evidências de que a dureza produza transtorno de ordem sanitária, ao contrário, de alguns estudos sinalizam para uma menor incidência de doenças cardíacas em áreas onde as águas apresentam maior dureza. No entanto, segundo a Portaria 518, de 25 de março de 2004 da ANVISA e Resolução n. ${ }^{\circ}$ 396/2008 do CONAMA, o limite máximo de dureza total em água potável é de $500 \mathrm{ppm}$, indicando, portanto, que neste requisito, a água analisada nos diferentes locais está de acordo com as normas citadas.

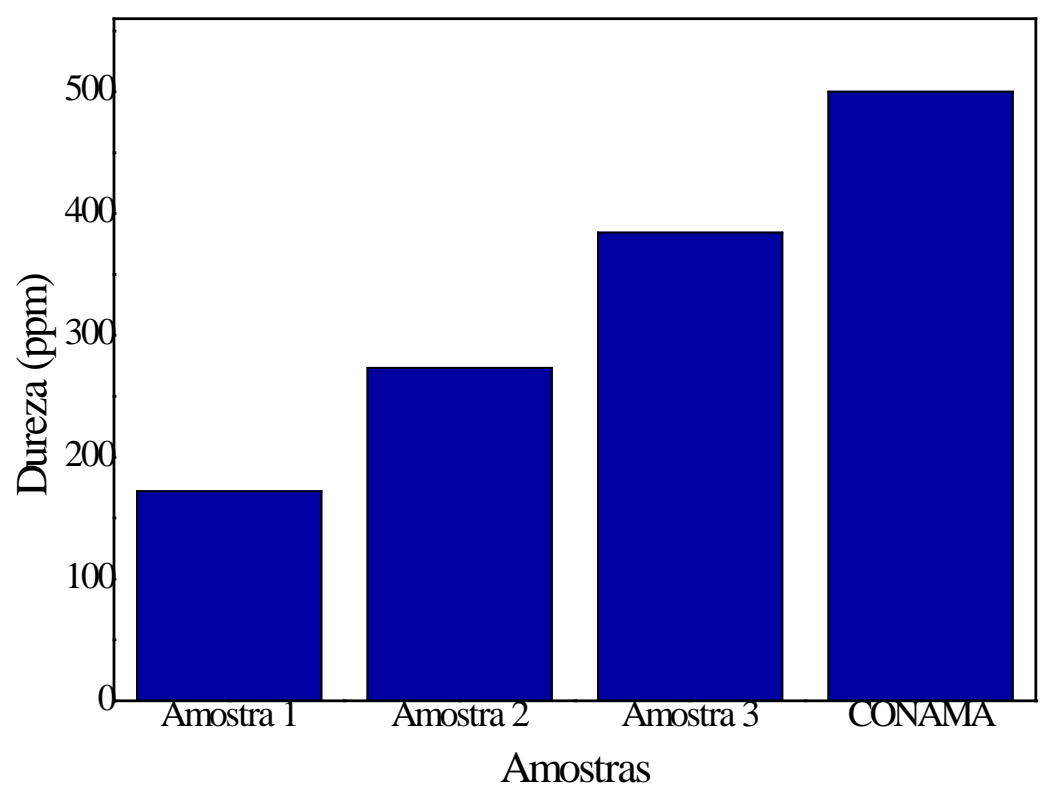

Gráfico 4- Resultados de dureza para as três amostras analisadas.

\section{CONCLUSÕES}

Algumas caraterísticas como sólidos dissolvidos, sulfato e nitrito estão de acordo com a resolução CONAMA n ${ }^{\circ}$ 396/2008, outras como o nitrato e o cloreto da amostra 2 demonstraram valores acima do permitido, evidenciando que os pontos 2 e 3 possuem um baixo serviço de esgotamento sanitário e uma densidade populacional maior. As características amônia, cloreto, alcalinidade, salinidade, fósforo e sulfato apresentaram um comportamento diferente, o que pode ser caracterizado pela densidade populacional e consequentemente uma maior produção de esgoto, o que resulta numa elevada concentração desses poluentes.

\section{Physico-chemical analysis of water from three underground wells in the city of Mossoro-RN}

Abstract: Water is a key resource for the existence of life. The planet Earth is flooded with water with a volume of approximately 1.4 billion $\mathrm{km}^{3}$ covering about $71 \%$ of Earth's surface. Nevertheless, many localities still lack access to potable water with appropriate characteristics to the needs of the consumer. The city of Mossoro-RN located in the northeastern semi-arid region, has $70 \%$ of its population supplied by water from underground wells. The quality of water for human consumption is of utmost importance and in general the natural waters of surfaces or underground, do not meet the recommended potability standards for consumption, requiring treatment and quality control. The lack of sanitation and above the acceptable exploitation are factors that contribute to the contamination of 
underground water sources, which are maximized by natural factors that reduce the availability of water as the drought affecting the region for five years. This study aimed to analyze groundwater samples collected at three different points of Mossoró-RN, and to compare the values obtained at each point between themselves and with the legislation in force, evaluating their quality and anthropogenic influences the location of these characteristics. The city of Mossoro-RN is located in western Rio Grande do Norte state, according to the IBGE census, in 2010, has a population of 259,886 habitants. Samples were collected from three wells with an approximate depth of 90 meters. Sample 1 was collected in the neighborhood New Bethany (Fields UNP), Sample 2 was collected in the center of Mossoró-RN (Rebouças Supermarket) and sample 3 was collected in the Alto de São Manoel (Mr. Residential Fausto William). The results showed that the water analyzed fall in class 3. Some characteristics such as dissolved solids, sulfate and nitrite to the serving CONAMA No. 396/2008 standard, others as chloride nitrate and specifically the sample 2 shown above permitted values, showing that the points 2 and 3 have a low sewage service and greater population density, which characterizes this fact.

Keywords: Quality; water; indicators.

\section{REFERÊNCI AS BI BLIOGRÁFICAS}

ALMEIDA, M. Geografia Global 2. São Paulo: Escala educacional, 1a ed. 2010.

APHA. Standard Methods for the examination of water and wasterwater. 21. ed. Washington, USA: American Public Health Association, 2005.

BRASI L, Ministério do Meio Ambiente. CONAMA - Conselho Nacional do Meio Ambiente.

Resolução № 396 de 03 de abril de 2008. Brasília, 2008.

BAIRD, C.; CANN, M. Química Ambiental. 4. ed. Porto Alegre: Bookman, 2011.

BECKER, H. Controle Analítico de Águas. Fortaleza-CE, Versão 4. p. 46, 2008.

BORSOI, Z. M. F. et al. Águas subterrâneas. 2002. Disponível em: <http://www.bndes.gov.br>. Acesso em: 26 set. 2015.

CETESB - COMPANHIA AMBIENTAL DO ESTADO DE SÃO PAULO. Variáveis de qualidade das águas. São Paulo, SP: CETESB, 2010.

CIRI LO, J. A. Políticas públicas de recursos hídricos para o semi-árido brasileiro. Estudos Avançados, v. 22, n. 63, p. 61-82, 2008.

FERREI RA, A. C.; ROCHA, L. C.; FIGUEIREDO, M. A. Análise do Índice De Qualidade de Água na Bacia do Córrego do Rio Acima, São João Del-Rei/MG. Revista Nacional de Gerenciamento de Cidades, v. 3, n. 15, p. 94-105, 2015.

GRASSI, M.T. As águas do planeta terra. Cadernos Temáticos de Química Nova na Escola, Edição Especial, p. 31-40, 2001.

IBGE. Cidades: Mossoró. Brasília: IBGE, 2014. Disponível em: <http://cidades.ibge.gov.br >. Acesso em: 16 de set. 2015.

INSTITUTO SOCIO AMBIENTAL: Água, o risco da escassez. Disponível em: <http://www.socioambiental.org> Acesso em: 27 set. 2015.

JACOBSEN, D.; et al. Biodiversity under threat in glacier-fed river systems. Nature Climate Change, v. 2, p. 361-364, 2012. 
MAFFEI, F. et al. Drinking water quality: an in vitro approach for the assessment of cytotoxic and genotoxic load in water sampled along distribution system. Environment I nternational, v. 35, p. 1053-1061, 2009.

PROGRAMA ÁGUA AZUL. Rede compartilhada de monitoramento da qualidade da água: monitoramento da qualidade das águas subterrâneas 1 orelatório parcial. Natal, março de 2011. Disponível em: < http://www.programaaguaazul.rn.gov.br >. Acesso em: 28 set. 2015.

RAHMAN, I. M. M. et al. Stagnant surface water bodies (SSWBs) as an alternative water resource for the Chittagong metropolitan area of Bangladesh: physicochemical characterization in terms of water quality indices. Environmental Monitoring and Assessment, v. 173, n. 1, p. 669-684, 2011.

REBOUÇAS, A.; GALÍZIA TUNDISI J.; BRAGA, B. Águas doces no Brasil: capital ecológico, uso e conservação. São Paulo: Instituto de Estudos Avançados, USP, 1999.

SAMPAIO, T. Q.; FEITOSA, F. A. C. Atuação governamental no abastecimento de água subterrânea no semiárido nordestino: o sistema SIAGAS e as possibilidades de revitalização de poços, In: Anais do $14^{\circ}$ Congresso Resag Enqualab 2014, São Paulo, 2014.

SILVA, M. A. O. Seca prolongada castiga população do campo na região do Seridó. Disponível em: <http://fcst.edu.br/site>. Acesso em: 26 set. 2015.

SOARES, T.M. et al. Destinação de águas residuais provenientes do processo de dessalinização por osmose reversa. Revista Brasileira de Engenharia Agrícola e Ambiental, v. 10, n. 3, p. 730737, 2006.

VAN VLIET, M. T. H. et al. Global river discharge and water temperature under climate change. Global Environmental Change, v. 23, n. 2, p. 450-464, 2013.

VÖRÖSMARTY, C. J.; et al. Global threats to human water security and river biodiversity. Nature, v. 467, n. 7315, p. 555-561, 2010. 